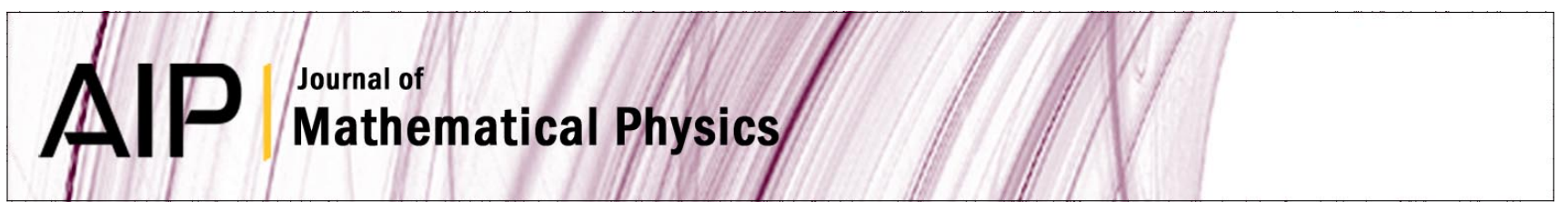

\title{
Chemically reacting mixtures in terms of degenerated parabolic setting
}

P. B. Mucha, M. Pokorný, and E. Zatorska

Citation: J. Math. Phys. 54, 071501 (2013); doi: 10.1063/1.4811564

View online: http://dx.doi.org/10.1063/1.4811564

View Table of Contents: http://jmp.aip.org/resource/1/JMAPAQ/v54/i7

Published by the AIP Publishing LLC.

\section{Additional information on J. Math. Phys.}

Journal Homepage: http://jmp.aip.org/

Journal Information: http://jmp.aip.org/about/about_the_journal

Top downloads: http://jmp.aip.org/features/most_downloaded

Information for Authors: http://jmp.aip.org/authors

\section{ADVERTISEMENT}

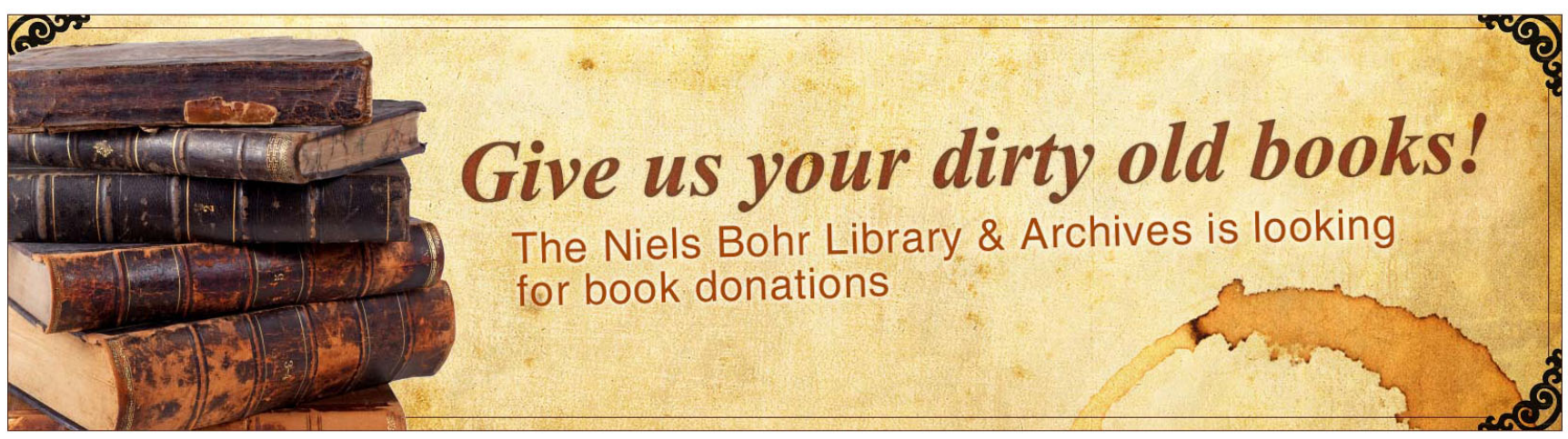




\title{
Chemically reacting mixtures in terms of degenerated parabolic setting
}

\author{
P. B. Mucha, ${ }^{1, a)}$ M. Pokorný, ${ }^{2, b)}$ and E. Zatorska ${ }^{1, c)}$ \\ ${ }^{1}$ Institute of Applied Mathematics and Mechanics, University of Warsaw, ul. Banacha 2, \\ 02-097 Warszawa, Poland \\ ${ }^{2}$ Charles University in Prague, Faculty of Mathematics and Physics, Mathematical Institute \\ of Charles University, Sokolovska 83, 18675 Praha, Czech Republic
}

(Received 25 July 2012; accepted 4 June 2013; published online 1 July 2013)

\begin{abstract}
The paper analyzes basic mathematical questions for a model of chemically reacting mixtures. We derive a model of several (finite) component compressible gas taking rigorously into account the thermodynamical regime. Mathematical description of the model leads to a degenerate parabolic equation with hyperbolic deviation. The thermodynamics implies that the diffusion terms are non-symmetric, not positively defined, and cross-diffusion effects must be strongly marked. The mathematical goal is to establish the existence of weak solutions globally in time for arbitrary number of reacting species. A key point is an entropy-like estimate showing possible renormalization of the system. (C)2013 AIP Publishing LLC. [http://dx.doi.org/10.1063/1.4811564]
\end{abstract}

\section{INTRODUCTION}

The mathematical study of models describing motion of chemically reacting gaseous mixtures is a very challenging area of research. This issue has been recently investigated in many aspects (Ref. 17) and there has been also considerable effort to prove the existence and to study the properties of solutions to associated systems of partial differential equations (PDEs) (Ref. 26). Such strong interest is caused by a variety of real word applications, especially in modern engineering, astrophysics, or medicine. Diversity of analytical methods developed to describe the mixtures is still insufficient to introduce the rigorous and uniform mathematical language based on the principles of fluid mechanics and thermodynamics. In fact, most of the nowadays available results cover only some simplified models (Refs. 8,11,12,19, and 28) (two species kinetics, irreversible reactions, 1D geometry, steady flow), while in reality, one has to deal with dozens or rather hundreds of species undergoing many reactions that are, in general, completely reversible, see Refs. 2, 10, and 16. Highly complex diffusion and cross-diffusion effects in these phenomena cause that the structure of the associated systems of PDEs ceases to be parabolic in the usual sense. First, the variable of the time differential operator differs from the variable of the space differential operator, second, the symmetrized matrix of diffusion coefficients is singular. Indeed, one can only hope for the coerciveness of the leading term on some physical hyperplane related to the alternating composition of the mixture.

In this paper, we intend to shed some light on this side of the problem, keeping the general thermodynamical framework (Ref. 16) and the regularity results for the binary mixture model considered in Ref. 29. Our result indicates a possibility of combination of Navier-Stokes system with a system of reaction-diffusion equations for the species. Due to the best of our knowledge, this issue, in the context of global-in-time existence of solutions and strong cross-diffusivity, is investigated here for the first time.

\footnotetext{
a) Electronic mail: p.mucha@mimuw.edu.pl

b) Electronic mail: pokorny @ karlin.mff.cuni.cz

c) Electronic mail: e.zatorska@ mimuw.edu.pl
} 
Following Ref. 16, we assume that a suitable description for the $n$-component $\varrho_{1}, \ldots, \varrho_{n}$ mixture of compressible gases is the set of reaction-diffusion equations

$$
\partial_{t} \varrho_{k}+\operatorname{div}\left(\varrho_{k} \mathbf{u}\right)+\operatorname{div}\left(\mathcal{F}_{k}\right)=\varrho \omega_{k}, \quad k=1, \ldots n,
$$

where $\varrho$ is the total mass, namely,

$$
\sum_{k=1}^{n} \varrho_{k}=\varrho,
$$

vector $\mathbf{u}$ is a given velocity which describes the transport of particles, $\omega_{k}=\omega_{k}\left(\varrho_{1}, \ldots, \varrho_{n}\right)$ determines the chemical reactions and $\mathcal{F}_{k}=\mathcal{F}_{k}\left(\varrho_{1}, \ldots, \varrho_{n}\right)$ denotes the diffusion flux.

The basic property of system (1) is that the sum of equations gives the time evolution of the total mass of the mixture, the so-called continuity equation

$$
\partial_{t} \varrho+\operatorname{div}(\varrho \mathbf{u})=0 .
$$

The main mathematical obstacle is the structure of diffusion fluxes. They form an elliptic operator, however not diagonal, even not symmetric, and first of all - degenerate. Thus any direct technique of renormalization of the system is not admissible as it is the case for the scalar system, Ref. 25. The only possibility is to employ the information concerning the entropy production (4); then we deal with the symmetric matrix $\mathbf{D}_{k l}=\mathbf{C}_{k l} l \varrho_{k}$, which is positive definite but only over a subspace of co-dimension 1 . Here we present our technique for a particular choice of thermodynamically admissible $\mathbf{C}_{k l}$ (17), cf. Ref. 16. It follows that the whole mathematical analysis should be done in terms of $\log p_{k}$, where $p_{k}$ denotes the partial pressure of the $k$ th component. This approach is effective, since it guarantees immediately that the densities of gas components will be non-negative. Using a relevant approximation we are allowed to obtain existence through the Galerkin approach, and then passing to subsequent limits we find the solution of the original problem. The compactness of the approximative sequence is guaranteed due to uniform $L \log L$ bounds and an extra information about the whole density $\rho$. The last fact allows to control the regularity of space derivatives of solutions. In other words, we extend the classical technique of Refs. 2 and 10 to the case of degenerate systems.

We discuss our model in terms of velocity, which in our case is given and is relatively smooth, particularly we are guaranteed that the continuity equation admits unique solutions. This last feature implies that the field should be such that $\operatorname{div} \boldsymbol{u} \in L^{2}\left(0, T ; L^{\infty}(\Omega)\right)$. On the other hand, the density is assumed to be regular, such smoothness one can find in Ref. 5 , where viscosity coefficients depend on the density. However, having regular enough velocity, smoothness of the density follows from this fact. Thus, our result raises a question if there is a possibility to relax assumptions on the velocity and the total initial density. The problem looks seriously, since it touches the fundamental difference between the scalar and system problems.

Recall that for the Navier-Stokes-type of compressible fluids models, the thermodynamical concept of entropy is of great importance (Refs. 13,20,22, and 23) as it provides majority of all available estimates. Following this path, one may expect the same for the multicomponent flows, still subject to a similar type of conservation laws (Refs. 3 and 14). Regrettably, it turns out that the approximation of the diffusion flux by the Fick law and the presence of the species concentrations in the state equation leads to difficulties in determining the sign of production of entropy associated with the diffusive process. Roughly speaking, one should be able to deduce directly from the form of $\mathcal{F}_{k}$ that

$$
-\int_{\Omega} \sum_{k=1}^{n} \frac{\mathcal{F}_{k}}{m_{k}} \cdot \nabla \log p_{k} \mathrm{~d} x \geq 0 .
$$

In consequence, to be physically consistent, one has to deal with a more general form of diffusion (chap. 7 of Ref. 16), leading to the mentioned degeneration in system (1).

For such kind of problems, the usual idea of applying the energy method to get a priori estimates cannot be used. Until now, only partial results for the well posedness of similar problems were available. The global in time solutions to system (1) coupled with momentum and energy equations 
and supplemented by physically relevant constitutive relations was established by Giovangigli (Ref. 16). He assumed, however, that the initial conditions are sufficiently close to an equilibrium state. The model describing one reversible, isothermal reaction was studied by Zatorska in Ref. 29, where the diffusion fluxes for two constituents $A$ and $B$ were taken in the form

$$
\begin{aligned}
& \mathcal{F}_{A}=-\left(\frac{\varrho_{B}}{\varrho m_{A}}+\frac{\varrho_{A}}{\varrho m_{B}}\right) \nabla \varrho_{A}-\frac{\varrho_{A}}{\varrho m_{B}} \nabla \varrho, \\
& \mathcal{F}_{B}=-\left(\frac{\varrho_{B}}{\varrho m_{A}}+\frac{\varrho_{A}}{\varrho m_{B}}\right) \nabla \varrho_{B}-\frac{\varrho_{B}}{\varrho m_{A}} \nabla \varrho .
\end{aligned}
$$

For this case, usual regularity of weak solutions to linear parabolic equations was possible to recover by additional estimate for the total density $\varrho$ found by Bresch-Desjardins (Ref. 6, see also Ref. 5). An outcome of Ref. 29 is that $\sqrt{\varrho} \in L^{\infty}\left(0, T ; H^{1}(\Omega)\right)$, where $\Omega$ is a periodic domain. This is precisely the assumption we make here, moreover the explicit choice of matrix $\mathbf{C}$ can be viewed as a generalization of the above to the case of arbitrary large, but finite, number of reacting species.

The systems of parabolic PDEs with strong cross-diffusion are also present in the population or the chemotaxis models (Refs. 9 and 15), for which the existence of certain Lyapunov functional often allows to introduce the entropy variables. Rewriting the system in terms of these variables usually leads to a symmetric and positive diffusion matrix, which may also help in proving non-negativity or even $L^{\infty}$ bounds. An overview of these methods can be found in Ref. 7.

To finish the introductory part, let us mention three possible interpretations of our result.

- The first one is the simplest case, namely, for $\boldsymbol{u} \equiv \mathbf{0}$. Then the density $\varrho$ is a given fixed function. The model takes into account just diffusion, neglecting the effects of transport. A relevant local-in-time existence result for such model has been obtained by Bothe (Ref. 4).

- The velocity field is given. The reactions have no influence on the speed of particles, they do not produce any internal force (pressure like force). Such model is admissible for "cold" reactions, where we do not observe any rapid changes of energy.

- System (1) is a part of the large model, where $\varrho, \varrho_{k}, \boldsymbol{u}$ are determined each other such as in Ref. 29. Then our result can be viewed as an auxiliary tool giving hints how to proceed with the full system.

\section{NOTATION AND MAIN RESULT}

We assume that $\Omega$ is a periodic box in $\mathbb{R}^{3}, \Omega=\mathbb{T}^{3}$ and we consider the following system of reaction-diffusion equations:

$$
\partial_{t} \varrho_{k}+\operatorname{div}\left(\varrho_{k} \mathbf{u}\right)+\operatorname{div}\left(\mathcal{F}_{k}\right)=\varrho \omega_{k}, \quad k=1, \ldots n
$$

with the initial conditions

$$
\varrho_{k}(0, \cdot)=\varrho_{k}^{0}(\cdot), \quad \varrho_{k}^{0} \geq 0, \quad \sum_{k=1}^{n} \int_{\Omega} \varrho_{k}^{0} \mathrm{~d} x=\int_{\Omega} \varrho^{0} \mathrm{~d} x=M_{0}
$$

Remark 1. From the point of view of the present work, there are no obstacles to assume the Neumann boundary conditions $\left.\mathcal{F}_{k} \cdot \mathbf{n}\right|_{\partial \Omega}=0$ together with the impermeability of boundary $\partial \Omega$, meaning $\left.\mathbf{u} \cdot \mathbf{n}\right|_{\partial \Omega}=0$. However, the higher regularity of the density that we assume in this paper has been so far proven only for the periodic domains (Ref. 29), so we stick to this restriction.

We now detail our assumptions on the diffusion flux $\mathcal{F}_{k}$ and the species production terms $\omega_{k}$, $k=1, \ldots, n$. 


\section{A. General conditions on $\mathcal{F}_{k}$}

In the case of isothermal chemical reactions and lack of external forces, the diffusion fluxes can be written in the following form:

$$
\mathcal{F}_{k}=-\mathbf{C}_{0} \sum_{l=1}^{n} \mathbf{C}_{k l} \mathbf{d}_{l}, \quad k=1, \ldots n
$$

where $\mathbf{C}_{0}, \mathbf{C}_{k l}$ are multicomponent flux diffusion coefficients and $\mathbf{d}_{k}=\left(d_{k}^{1}, d_{k}^{2}, d_{k}^{3}\right)$ is the species $k$ diffusion force

$$
d_{k}^{i}=\nabla_{x_{i}}\left(\frac{p_{k}}{\pi}\right)+\left(\frac{p_{k}}{\pi}-\frac{\varrho_{k}}{\varrho}\right) \nabla_{x_{i}} \log \pi
$$

In the above formula $\pi=\pi\left(\varrho_{1}, \ldots, \varrho_{n}\right)$ denotes the internal pressure of the mixture which is determined through the Boyle law

$$
\pi\left(\varrho_{1}, \ldots, \varrho_{n}\right)=\sum_{k=1}^{n} p_{k}\left(\varrho_{k}\right)=\sum_{k=1}^{n} \frac{\varrho_{k}}{m_{k}},
$$

where $m_{k}$ is the molar mass of the species $k$.

The main properties of the flux diffusion matrix $\mathbf{C}=\left(\mathbf{C}_{k, l}\right)_{k, l=1}^{n}$ are the following (see Ref. 16):

$$
\mathbf{C Y}=\mathcal{Y} \mathbf{C}^{T}, \quad N(\mathbf{C})=\mathbb{R} \vec{Y}, \quad R(\mathbf{C})=\vec{U}^{\perp},
$$

where $\mathcal{Y}=\operatorname{diag}\left(Y_{1}, \ldots, Y_{n}\right)$ is the diagonal matrix of species mass fractions $Y_{k}$ given by $Y_{k}=\frac{\varrho_{k}}{\varrho}$, $k=1, \ldots, n, N(\mathbf{C})$ is the nullspace of matrix $\mathbf{C}$ and by $R(\mathbf{C})$ we denote its range; $\vec{Y}=\left(Y_{1}, \ldots, Y_{n}\right)^{T}$ is the mass fraction vector, $\vec{U}=(1, \ldots, 1)^{T}$ and $\vec{U}^{\perp}$ are the orthogonal complements of $\mathbb{R} \vec{U}$.

Another important condition on C, postulated, for example, by Waldmann (Ref. 27), is that wherever it can be defined, the matrix

$$
\mathbf{D}_{k l}=\frac{\mathbf{C}_{k l}}{\varrho_{k}}, \quad k, l=1, \ldots, n
$$

is symmetric and positive definite over the physical hyperplane $\vec{U}^{\perp}$, which corresponds to the positivity of entropy production rate associated with diffusive process. For more details on evaluation of $\mathbf{C}$ from the kinetic theory of gases as well as its mathematical properties, we refer the reader to the textbook of Giovangigli (chaps. 4 and 7 of Ref. 16, and the references therein).

\section{B. Species production rates}

For the isothermal reactions, the species production rates are functions of the species mass fractions only. We will additionally assume that they are Lipschitz continuous with respect to $\varrho_{1}, \ldots, \varrho_{n}$ and that there exist positive constants $\underline{\omega}$ and $\bar{\omega}$ such that

$$
-\underline{\omega} \leq \omega_{k}\left(\varrho_{1}, \ldots, \varrho_{n}\right) \leq \bar{\omega}, \quad \text { for all } \quad 0 \leq Y_{k} \leq 1, \quad k=1, \ldots, n ;
$$

moreover, we suppose that

$$
\omega_{k}\left(Y_{1}, \ldots, Y_{n}\right) \geq 0 \quad \text { whenever } Y_{k}=0 .
$$

We also anticipate the mass constraint between the chemical source terms

$$
\sum_{k=1}^{n} \omega_{k}=0
$$

Another restriction that we postulate for chemical sources is dictated by the second law of thermodynamics, which asserts that the entropy production associated with any admissible chemical reaction 
is non-negative. In particular, $\omega_{k}$ must enjoy the following condition:

$$
-\int_{\Omega} \sum_{k=1}^{n} \frac{g_{k} \varrho \omega_{k}}{\vartheta} \mathrm{d} x \geq 0
$$

where $\vartheta$ is the absolute temperature and $g_{k}$ are the Gibbs functions of species. For fixed positive $\vartheta$ and equal constant-pressure specific heats for all the species, this condition may be translated into the following one (see chap. 2 of Ref. 16),

$$
\int_{\Omega} \sum_{k=1}^{n} \frac{\log p_{k} \omega_{k} \varrho}{m_{k}} \mathrm{~d} x \leq c,
$$

which allows us to control the source term in the main estimate (29). Here and subsequently $c$ denotes a constant that may differ throughout the paper and, if it is not marked otherwise, depends only on the data.

Remark 2. From (7) and (9) it follows that $\overrightarrow{\mathcal{F}}=\left(\mathcal{F}_{1}, \ldots, \mathcal{F}_{n}\right)^{T} \in R(\mathbf{C})=\vec{U}^{\perp}$, therefore, taking the scalar product between $\overrightarrow{\mathcal{F}}$ and $\vec{U}$ it can be deduced that

$$
\sum_{k=1}^{n} \mathcal{F}_{k}=0
$$

which together with (13) leads to the continuity equation for $\varrho$,

$$
\partial_{t} \varrho+\operatorname{div}(\varrho \mathbf{u})=0, \quad \varrho^{0}=\sum_{k=1}^{n} \varrho_{k}^{0} .
$$

In particular, the total mass of the mixture is conserved

$$
\int_{\Omega} \varrho(t) \mathrm{d} x=M_{0}, \quad \text { for a.a. } t \in(0, T) .
$$

\section{Explicit form of diffusion matrix C}

Since the chemical reactions considered in this paper may be completely reversible, the symmetric role is given to all of the species. Therefore, to fix the idea, we shall concentrate on the following explicit form of $\mathbf{C}$ :

$$
\mathbf{C}=\left(\begin{array}{cccc}
Z_{1} & -Y_{1} & \ldots & -Y_{1} \\
-Y_{2} & Z_{2} & \ldots & -Y_{2} \\
\vdots & \vdots & \ddots & \vdots \\
-Y_{n} & -Y_{n} & \ldots & Z_{n}
\end{array}\right)
$$

where $Z_{k}=\sum_{\substack{i=1 \\ i \neq k}}^{n} Y_{i}$. For the sake of simplicity, we assume that $\mathbf{C}_{0}=\pi$.

Remark 3. Note that the matrix $\mathbf{C}$ is singular since $\mathbf{C} \vec{Y}=0$ and is not symmetric in general.

Remark 4. It is easy to check that by the expressions for the diffusion forces (8) and the properties of $\mathbf{C}$, one can rewrite (7) into the following form:

$$
\mathcal{F}_{k}=-\left(\nabla p_{k}-Y_{k} \nabla \pi\right)=-\sum_{l=1}^{n} \mathbf{C}_{k l} \nabla p_{l}
$$




\section{Main result}

The main result of this paper is the following.

Theorem 1. Let $\varrho$ be a sufficiently smooth solution of (16) such that $\varrho$ is bounded in $L^{2}\left(0, T ; W^{1,2}(\Omega)\right)$. Moreover, let $0<\inf _{\Omega} \varrho^{0} \leq \sup _{\Omega} \varrho^{0}<\infty$. Let $\mathbf{u} \in L^{\infty}((0, T) \times \Omega)$ be fixed such that $\operatorname{div} \mathbf{u} \in L^{2}\left((0, T) ; L^{\infty}(\Omega)\right)$. Assuming (11)-(17) problem [(5) and (6))] admits a global in time weak solution, such that

$$
\varrho_{k} \geq 0 \quad \text { a.e. in }(0, T) \times \Omega, \quad \sum_{k=1}^{n} \int_{\Omega} \varrho_{k}(t) \mathrm{d} x=M_{0} .
$$

Furthermore, the following regularity properties hold:

$$
\varrho_{k} \in C\left([0, T] ; L \log L_{\text {weak }^{*}}(\Omega)\right) \quad \text { and } \quad \nabla \sqrt{\varrho_{k}} \in L^{2}((0, T) \times \Omega) .
$$

Remark 5. To maintain consistency with the existence results for a class of models with densitydependent viscosity coefficients (Refs. 5,21, and 29) one should rather work with $\sqrt{\varrho}$ instead of $\varrho$, since it is only possible to show integrability of $\nabla \sqrt{\varrho}$. Note that we actually need that $\sqrt{\varrho} \in L^{2}\left(0, T ; W^{1,2}(\Omega)\right)$ but due to assumptions on $\boldsymbol{u}$ and $\varrho^{0}$ it is equivalent with the assumption in the theorem.

Remark 6. Note that assuming $\boldsymbol{u}$ much more regular, we would be able to recover the regularity of the density $\varrho$ required in Theorem 1. Note, however, that in practical applications, except for very special situations, such a regularity is not possible to obtain.

The rest of this article is devoted to the proof of this theorem. The strategy is to add the standard regularization terms and then to employ the Galerkin method for the system rewritten in terms of so-called entropy variables.

\section{EXISTENCE OF SOLUTIONS}

\section{A. Galerkin approximation}

Our aim is to construct the weak solution to the semi-linear parabolic problem

$$
\begin{gathered}
\left(\delta+\mathrm{e}^{r_{k}}\right) \partial_{t} r_{k}+\operatorname{div}\left(\mathrm{e}^{r_{k}} \mathbf{u}\right)-\operatorname{div}\left(\left(\delta+\varepsilon \mathrm{e}^{r_{k}}\right) \nabla r_{k}\right)+\frac{\operatorname{div} \mathcal{F}_{k}}{m_{k}}=\frac{\varrho \omega_{k}}{m_{k}}, \\
r_{k}(0, x)=r_{k}^{0},
\end{gathered}
$$

for every $k=1, \ldots, n,(t, x) \in[0, T] \times \Omega$, and for any given, smooth (as in Theorem 1) vector u. To this purpose, we will employ the Galerkin technique. We denote by $N$ the (finite) dimension of the approximation. The aim (achieved in Subsection III B) will be to pass with $N \rightarrow \infty$. More precisely, we assume that $r_{k, N}$ has the following structure:

$$
r_{k, N}=\sum_{i=1}^{N} a_{k, N}^{i}(t) h_{i}(x),
$$

where the functions $\left\{h_{i}\right\}_{i \in \mathbb{N}}$ form an orthogonal basis of the Hilbert space $W^{1,2}(\Omega)$, they are smooth and orthonormal with respect to the scalar product $(\cdot, \cdot)$ in $L^{2}(\Omega)$. We look for the coefficients $a_{k, N}^{i}(t), t \in[0, T], k=1, \ldots, n, i=1, \ldots, N$ such that

$$
a_{k, N}^{i}(0)=\left(r_{k}^{0}, h_{i}\right)
$$


and the following equality is satisfied:

$$
\begin{aligned}
\int_{\Omega} \partial_{t}\left(\delta r_{k, N}\right. & \left.+\mathrm{e}^{r_{k, N}}\right) h_{l} \mathrm{~d} x \\
& =-\int_{\Omega} \operatorname{div}\left(\mathrm{e}^{r_{k, N}} \mathbf{u}-\left(\delta+\varepsilon \mathrm{e}^{r_{k, N}}\right) \nabla r_{k, N}+\frac{\mathcal{F}_{k, N}}{m_{k}}\right) h_{l} \mathrm{~d} x+\int_{\Omega} \frac{\varrho_{N} \omega_{k}}{m_{k}} h_{l} \mathrm{~d} x,
\end{aligned}
$$

for any $l=1, \ldots, N$. Here,

$$
\varrho_{N}=\sum_{k=1}^{n} m_{k} \mathrm{e}^{r_{k, N}}
$$

and $\mathcal{F}_{k, N}$ is given in (26) below. We have the following.

Theorem 2. For any $N \in \mathbb{N}$ there exist uniquely determined functions $r_{1, N}, \ldots, r_{n, N}$ of the form (20) satisfying (21) and (22). Moreover, there exists a constant $c$ depending only on $T$ and the initial data, such that

$$
\begin{aligned}
& \sqrt{\delta}\left\|r_{k, N}\right\|_{L^{\infty}\left(0, T ; L^{2}(\Omega)\right)}+\left\|\mathrm{e}^{r_{k, N}} r_{k, N}\right\|_{L^{\infty}\left(0, T ; L^{1}(\Omega)\right)} \\
& +\sqrt{\delta}\left\|\nabla r_{k, N}\right\|_{L^{2}((0, T) \times \Omega)}+\sqrt{\varepsilon}\left\|\nabla \sqrt{\mathrm{e}^{r_{k, N}}}\right\|_{L^{2}((0, T) \times \Omega)}+\sum_{k=1}^{n}\left\|\frac{\mathcal{F}_{k, N}}{\sqrt{m_{k} \mathrm{e}^{r_{k, N}}}}\right\|_{L^{2}((0, T) \times \Omega)} \leq c .
\end{aligned}
$$

Proof. This task is equivalent with solving the set of $N$ ordinary differential equations (ODEs) corresponding to each of $n$ equations of the system (22),

$$
\delta \dot{a}_{k, N}^{l}(t)+\sum_{i=1}^{N} \dot{a}_{k, N}^{i}\left(\mathrm{e}^{r_{k, N}} h_{i}, h_{l}\right)=-\mathbb{X}_{k, N}\left(r_{k, N}, h_{l}\right)+\left(\frac{\varrho_{N} \omega_{k}}{m_{k}}, h_{l}\right),
$$

with the initial conditions given by (21). In the above formula, $\varrho_{N}$ is given by (23), $\dot{a}(t)$ is time derivative of $a(t)$, and

$$
\begin{gathered}
\mathbb{X}_{k, N}\left(r_{k, N}, h_{l}\right)=\int_{\Omega} \operatorname{div}\left(\mathrm{e}^{r_{k, N}} \mathbf{u}-\left(\delta+\varepsilon \mathrm{e}^{r_{k, N}}\right) \nabla r_{k, N}+\frac{\mathcal{F}_{k, N}}{m_{k}}\right) h_{l} \mathrm{~d} x, \\
\mathcal{F}_{k, N}=-\left(\nabla \mathrm{e}^{r_{k, N}}-Y_{k, N} \sum_{j=1}^{n} \mathrm{e}^{r_{j, N}} \nabla r_{j, N}\right)=-\sum_{j=1}^{n} \mathbf{C}_{k j, N} \mathrm{e}^{r_{j, N}} \nabla r_{j, N}
\end{gathered}
$$

The matrix $\mathbf{C}_{N}$ is given by

$$
\mathbf{C}_{N}=\left(\begin{array}{cccc}
Z_{1, N} & -Y_{1, N} & \ldots & -Y_{1, N} \\
-Y_{2, N} & Z_{2, N} & \ldots & -Y_{2, N} \\
\vdots & \vdots & \ddots & \vdots \\
-Y_{n, N} & -Y_{n, N} & \ldots & Z_{n, N}
\end{array}\right)
$$

where

$$
Y_{j, N}=\frac{m_{j} \mathrm{e}^{r_{j, N}}}{\varrho_{N}} \quad \text { and } \quad Z_{k, N}=\sum_{\substack{i=1 \\ i \neq k}}^{n} Y_{i, N}
$$

Observe that since the matrix $\mathbb{X}_{k, N}$ involves all $n$ functions $r_{k, N}$, we should solve the system of $N \cdot n$ ODEs simultaneously. To this purpose, we rewrite system (25) using the vector $A_{k, N}(t)$ 
$=\left(a_{k, N}^{1}(t), \ldots, a_{k, N}^{N}(t)\right)^{T}$ into the following form:

$$
\left(\delta \mathbb{I}+\mathbb{B}_{k, N}(t)\right) \dot{A}_{k, N}(t)=-\mathbb{X}_{k, N}\left(r_{k, N}, h^{N}\right)+\left(\frac{\varrho_{N} \omega_{k}}{m_{k}}, h^{N}\right), \quad k=1, \ldots, n,
$$

where $\mathbb{I}$ is the identity matrix,

$$
\begin{gathered}
\left(\mathbb{B}_{k, N}\right)_{i j}=\int_{\Omega} \mathrm{e}^{r_{k, N}} h_{i} h_{j} \mathrm{~d} x, \\
\mathbb{X}_{k, N}\left(r_{k, N}, h^{N}\right)=\left(\mathbb{X}_{k, N}\left(r_{k, N}, h_{1}\right), \ldots, \mathbb{X}_{k, N}\left(r_{k, N}, h_{N}\right)\right)^{T},
\end{gathered}
$$

and

$$
\left(\frac{\varrho \omega_{k}}{m_{k}}, h^{N}\right)=\left(\left(\frac{\varrho \omega_{k}}{m_{k}}, h_{1}\right), \ldots, \quad\left(\frac{\varrho \omega_{k}}{m_{k}}, h_{N}\right)\right)^{T} .
$$

It is easy to see that the matrix $\delta \mathbb{I}+\mathbb{B}_{k, N}(t)$ is invertible for any $\delta>0$; indeed $\mathrm{e}^{r_{k, N}}$ is a non-negative function, thus the time-dependent bilinear form

$$
\mathbb{B}_{k, N}\left[h_{i}, h_{j} ; t\right]=\left(\mathbb{B}_{k, N}\right)_{i j}=\int_{\Omega} \mathrm{e}^{r_{k, N}} h_{i} h_{j} \mathrm{~d} x
$$

is symmetric and positive-semidefinite. Next, using the following property of the block diagonal matrixes:

$$
\left(\begin{array}{cccc}
\mathbb{A}_{1, N} & 0 & \ldots & 0 \\
0 & \mathbb{A}_{2, N} & \ldots & 0 \\
\vdots & \vdots & \ddots & \vdots \\
0 & 0 & \ldots & \mathbb{A}_{n, N}
\end{array}\right)^{-1}=\left(\begin{array}{cccc}
\mathbb{A}_{1, N}^{-1} & 0 & \ldots & 0 \\
0 & \mathbb{A}_{2, N}^{-1} & \ldots & 0 \\
\vdots & \vdots & \ddots & \vdots \\
0 & 0 & \ldots & \mathbb{A}_{n, N}^{-1}
\end{array}\right),
$$

we invert the $(n \cdot N) \times(n \cdot N)$ matrix that stands in front of the time derivative of system (27) and therefore, problem (25) can be replaced by the following one:

$$
\dot{A}_{k, N}(t)=-\left(\delta \mathbb{I}+\mathbb{B}_{k, N}(t)\right)^{-1} \mathbb{X}_{k, N}\left(r_{k, N}, h^{N}\right)+(\delta \mathbb{I}+\mathbb{B}(t))^{-1}\left(\frac{\varrho_{N} \omega_{k}}{m_{k}}, h^{N}\right) .
$$

Before we apply the classical result on solvability of the ODE system we check whether the right hand side of (28) is Lipschitz with respect to $a_{k, l}(t)$ for all $k=1, \ldots, n, l=1, \ldots, N$. This is true on account of the fact that a finite composition of the polynomials and the exponent functions is a Lipschitz function, at least locally with respect to time. Therefore, for sufficiently short time interval $[0, \tau)$ there exists uniformly continuous (with respect to time) solution to problem (25).

In order to obtain the global in time solution any a priori estimate on $r_{k, N}$ is needed, because on a finite-dimensional space all norms are equivalent. To this end, we use in (22) the test function $r_{k, N}$ (we multiply each of the equations by $a_{k, N}^{l}(t)$ first, and then we sum them with respect to $l=1, \ldots, N)$. Integrating by parts we obtain the following equality:

$$
\begin{aligned}
\sum_{k=1}^{n} \frac{d}{d t} \int_{\Omega}\left(\delta \frac{r_{k, N}^{2}}{2}+\mathrm{e}^{r_{k, N}} r_{k, N}-\mathrm{e}^{r_{k, N}}\right) & \mathrm{d} x+\sum_{k=1}^{n} \int_{\Omega}\left(\left(\delta+\varepsilon \mathrm{e}^{r_{k, N}}\right)\left|\nabla r_{k, N}\right|^{2}-\frac{\mathcal{F}_{k, N}}{m_{k}} \nabla r_{k, N}\right) \mathrm{d} x \\
= & -\sum_{k=1}^{n} \int_{\Omega} \mathrm{e}^{r_{k, N}} \operatorname{div} \mathbf{u} \mathrm{d} x+\sum_{k=1}^{n} \int_{\Omega} \frac{\varrho_{N} \omega_{k}}{m_{k}} r_{k, N} \mathrm{~d} x
\end{aligned}
$$

The only problematic term on the left hand side is the last one. However, since $\sum_{k=1}^{n} \mathcal{F}_{k, N}=0-$ see (15) - we can write

$$
\sum_{k=1}^{n}\left(\frac{\mathcal{F}_{k, N}}{m_{k} \mathrm{e}^{r_{k, N}}} Y_{k, N} \sum_{j=1}^{n} \mathrm{e}^{r_{j, N}} \nabla r_{j, N}\right)=0
$$


and therefore the last term on the left hand side of (29) may be written as follows:

$$
\begin{aligned}
-\sum_{k=1}^{n} \frac{\mathcal{F}_{k, N}}{m_{k}} \nabla r_{k, N} & =-\sum_{k=1}^{n} \frac{\mathcal{F}_{k, N}}{m_{k} \mathrm{e}^{r_{k, N}}} \nabla \mathrm{e}^{r_{k, N}}= \\
& -\sum_{k=1}^{n} \frac{\mathcal{F}_{k, N}}{m_{k} \mathrm{e}^{r_{k, N}}}\left(\nabla \mathrm{e}^{r_{k, N}}-Y_{k, N} \sum_{j=1}^{n} \mathrm{e}^{r_{j, N}} \nabla r_{j, N}\right)=\sum_{k=1}^{n} \frac{\mathcal{F}_{k, N}^{2}}{m_{k} \mathrm{e}^{r_{k, N}} \geq 0 .}
\end{aligned}
$$

Thus, to get the estimates one only needs to control the right hand side of (29). Substituting in (14) $p_{k}=\mathrm{e}^{r_{k, N}}$ and $\varrho=\varrho_{N}$ we deduce that the last term on the right hand side of (29) is bounded

$$
\sum_{k=1}^{n} \int_{\Omega} \frac{\varrho_{N} \omega_{k}}{m_{k}} r_{k, N} \mathrm{~d} x \leq c .
$$

For the remaining one, we have

$$
\sum_{k=1}^{n} \int_{\Omega}\left|\mathrm{e}^{r_{k, N}} \operatorname{div} \mathbf{u}\right| \mathrm{d} x \leq\|\operatorname{div} \mathbf{u}\|_{\infty} \sum_{k=1}^{n} \int_{\Omega} \mathrm{e}^{r_{k, N}} \mathrm{~d} x .
$$

We are now at the position to deduce that (29) implies

$$
\begin{aligned}
& \sum_{k=1}^{n} \frac{d}{d t}\left(\left\|\delta r_{k, N}^{2}+\mathrm{e}^{r_{k, N}} r_{k, N}-\mathrm{e}^{r_{k, N}}\right\|_{L^{1}(\Omega)}\right)+ \\
& \sum_{k=1}^{n}\left\{\delta\left\|\nabla r_{k, N}\right\|_{L^{2}(\Omega)}^{2}+\varepsilon\left\|\nabla \sqrt{\mathrm{e}^{r_{k, N}}}\right\|_{L^{2}(\Omega)}^{2}+\left\|\frac{\mathcal{F}_{k, N}}{\sqrt{m_{k} \mathrm{e}^{r_{k, N}}}}\right\|_{L^{2}(\Omega)}^{2}\right\} \\
& \leq c \sum_{k=1}^{n}\left(1+\|\operatorname{div} \mathbf{u}\|_{L^{\infty}(\Omega)}\left\|\mathrm{e}^{r_{k, N}}\right\|_{L^{1}(\Omega)}\right) .
\end{aligned}
$$

Because the term $r_{k, N}^{2}$ is non-negative and $\mathrm{e}^{r_{k, N}} r_{k, N}$ is bounded from below, $\operatorname{div} \mathbf{u} \in L^{2}\left(0, T ; L^{\infty}(\Omega)\right)$, we get using the Gronwall argument estimate (24). As was already announced, this estimate allows us to repeat the procedure described before in order to extend the solution to the whole time interval $[0, T]$.

Although the above construction corresponds only to particular projection of the original problem it is clear that the final estimate is completely independent of $N$. This is the key argument in the limit passage; derivation of the other uniform estimates is a purpose of Subsection III B.

\section{B. Passage to the limit $N \rightarrow \infty$}

Our next goal is to derive bounds uniform with respect to $N$ for fixed $\delta, \varepsilon>0$ and $\mathbf{u}$ as in Theorem 1. We have already mentioned that estimate (24) obtained in Subsection III A does not depend on the dimension of Galerkin approximations. In particular, we have that

$$
\left|\nabla \mathrm{e}^{r_{k, N}}\right| \leq 2\left|\nabla \sqrt{\mathrm{e}^{r_{k, N}}}\right| \sqrt{\mathrm{e}^{r_{k, N}}}
$$

is bounded in $L^{2}\left(0, T ; L^{1}(\Omega)\right)$, therefore, by the Sobolev imbedding, $\mathrm{e}^{r_{k, N}}$ is bounded in $L^{2}\left(0, T ; L^{\frac{3}{2}}(\Omega)\right)$. Returning to $(32)$ we get

$$
\left\|\nabla \mathrm{e}^{r_{k, N}}\right\|_{L^{\frac{4}{3}}\left(0, T ; L^{\frac{6}{5}}(\Omega)\right)} \leq c ;
$$

using once more the Sobolev imbedding theorem and the bound in $L^{\infty}\left(0, T ; L^{1}(\Omega)\right)$ we end up with

$$
\left\|\mathrm{e}^{r_{k, N}}\right\|_{L^{\frac{5}{3}}((0, T) \times \Omega)} \leq c(\varepsilon) .
$$


Having this, we can return to (32) to deduce

$$
\left\|\nabla \mathrm{e}^{r_{k, N}}\right\|_{L^{\frac{5}{4}}((0, T) \times \Omega)} \leq c(\varepsilon) .
$$

Apart from that, the limit passage requires also some further estimates providing compactness with respect to time.

Lemma 3. There exists a constant $c$ depending on the initial data, $T$, and the parameter $\varepsilon$ such that

$$
\delta\left\|\partial_{t} r_{k, N}\right\|_{L^{\frac{5}{4}}\left(0, T ; W^{-1, \frac{5}{4}}(\Omega)\right)} \leq c .
$$

Proof. We take any $\phi \in W^{1,5}(\Omega) \subset W^{1,2}(\Omega)$ such that $\|\phi\|_{W^{1,5}(\Omega)} \leq 1$ and decompose it into $\phi$ $=\phi_{1}+\phi_{2}$, where $\phi_{1}$ is an orthogonal projection of $\phi$ (with respect to the scalar product induced by the norm of the space $L^{2}(\Omega)$ ) onto the subspace spanned by the vectors $\left\{h_{1}, \ldots, h_{N}\right\}$. Using $\phi_{1}$ as a test function in (22) we show that

$$
\begin{aligned}
& \int_{\Omega} \partial_{t}\left(\delta r_{k, N}+\mathrm{e}^{r_{k, N}}\right) \phi_{1} \mathrm{~d} x=\int_{\Omega}\left(\mathrm{e}^{r_{k, N}} \mathbf{u}-\left(\delta+\varepsilon \mathrm{e}^{r_{k, N}}\right) \nabla r_{k, N}+\frac{\mathcal{F}_{k, N}}{m_{k}}\right) \cdot \nabla \phi_{1} \mathrm{~d} x+\int_{\Omega} \frac{\varrho_{N} \omega_{k}}{m_{k}} \phi_{1} \mathrm{~d} x \\
& \quad \leq \sum_{k=1}^{n}\left(\|\mathbf{u}\|_{L^{\infty}(\Omega)}\left\|\mathrm{e}^{r_{k, N}}\right\|_{L^{\frac{5}{4}(\Omega)}}+\delta\left\|\nabla r_{k, N}\right\|_{L^{\frac{5}{4}}(\Omega)}+\varepsilon\left\|\nabla \mathrm{e}^{r_{k, N}}\right\|_{L^{\frac{5}{4}}(\Omega)}\right)\left\|\phi_{1}\right\|_{W^{1,5}(\Omega)} \\
& +c \sum_{k=1}^{n}\left\|\frac{\mathcal{F}_{k, N}}{\sqrt{m_{k} \mathrm{e}^{r_{k, N}}}}\right\|_{L^{2}(\Omega)}\left\|\sqrt{\mathrm{e}^{r_{k, N}}}\right\|_{L^{\frac{10}{3}}(\Omega)}\left\|\phi_{1}\right\|_{W^{1,5}(\Omega)}+c \sum_{k=1}^{n}\left\|\mathrm{e}^{r_{k, N}}\right\|_{L^{\frac{5}{4}}(\Omega)}\left\|\phi_{1}\right\|_{W^{1,5}(\Omega)} \cdot
\end{aligned}
$$

Then we have

$$
\begin{aligned}
\left\|\partial_{t} r_{k, N}(t, \cdot)\right\|_{W^{-1, \frac{5}{4}(\Omega)}}= & \sup _{\phi \in W^{1,5}(\Omega) ;\|\phi\| \leq 1}\left|\int_{\Omega} \partial_{t} r_{k, N}(t, \cdot) \phi \mathrm{d} x\right| \\
& =\sup _{\phi \in W^{1,5}(\Omega) ;\|\phi\| \leq 1}\left|\int_{\Omega} \partial_{t} r_{k, N}(t, \cdot) \phi_{1} \mathrm{~d} x\right|=\int_{\Omega}\left|\partial_{t} r_{k, N}(t, \cdot) \varphi_{1}\right| \mathrm{d} x
\end{aligned}
$$

for some $\varphi_{1} \in W_{0}^{1,5}(\Omega) \cap \operatorname{Lin}\left\{h_{1}, \ldots, h_{N}\right\}$. Hence

$$
\left\|\partial_{t} r_{k, N}(t, \cdot)\right\|_{W^{-1, \frac{5}{4}(\Omega)}} \leq \sup _{\phi \in W^{1,5}(\Omega) \cap \operatorname{Lin}\left\{h_{1} \ldots h_{N}\right\} ;\|\phi\| \leq 1} \frac{1}{\delta}\left|\int_{\Omega}\left(\delta+\mathrm{e}^{r_{k, N}(t, \cdot)}\right) \partial_{t} r_{k, N}(t, \cdot) \phi \mathrm{d} x\right|
$$

and due to estimate (37) we end up with

$$
\left\|\partial_{t} r_{k, N}\right\|_{L^{\frac{5}{4}}\left(0, T ; W^{\left.-1, \frac{5}{4}(\Omega)\right)}\right.} \leq \frac{c(\varepsilon)}{\delta} .
$$

Our goal in the remaining part of this subsection is to examine the limit for $N \rightarrow \infty$. The above lemma allows us to apply the Aubin-Lions lemma in order to extract the subsequences which satisfy (19) in the limit. Indeed, for the sequence $r_{k, N}$ we deduce from (24) that it is possible to extract a subsequence such that

$$
\begin{gathered}
r_{k, N} \rightarrow r_{k} \quad \text { weakly* in } L^{\infty}\left(0, T ; L^{2}(\Omega)\right), \\
\nabla r_{k, N} \rightarrow \nabla r_{k} \quad \text { weakly in } L^{2}((0, T) \times \Omega), \\
\partial_{t} r_{k, N} \rightarrow \partial_{t} r_{k} \quad \text { weakly in } L^{\frac{5}{4}}\left(0, T ; W^{-1, \frac{5}{4}}(\Omega)\right), \\
r_{k, N} \rightarrow r_{k} \quad \text { strongly in } L^{2}\left(0, T ; L^{p}(\Omega)\right), p<6 ;
\end{gathered}
$$


in particular, there exists a subsequence $r_{k, N}$ which converges to $r_{k}$ a.e. on $(0, T) \times \Omega$. This, together with (36) and the boundedness of space gradient of $\mathrm{e}^{r_{k, N}}$ implies

$$
\begin{gathered}
\nabla \mathrm{e}^{r_{k, N}} \rightarrow \nabla \mathrm{e}^{r_{k}} \quad \text { weakly in } L^{2}\left(0, T ; L^{1}(\Omega)\right) \cap L^{\frac{5}{4}}((0, T) \times \Omega), \\
\mathrm{e}^{r_{k, N}} \rightarrow \mathrm{e}^{r_{k}} \quad \text { strongly in } L^{q}((0, T) \times \Omega), q<\frac{5}{3},
\end{gathered}
$$

at least for a chosen subsequence.

Next, we recall basics facts from the theory of Orlicz spaces. For more details as well as proofs of results below see, e.g., chap. 3 of Ref. 18 or chap. 8 of Ref. 1.

For the following pair of complementary Young functions:

$$
\Phi(t)=\mathrm{e}^{t}-1 ; \quad \Psi= \begin{cases}0 & \text { for } 0 \leq t<1, \\ t(\log t-1) & \text { for } t \geq 1 .\end{cases}
$$

we consider the Orlicz spaces $L_{\Phi}(\Omega), L_{\Psi}(\Omega):=L \log L(\Omega)$ and the space $E_{\Phi}(\Omega)$, which is the closure of bounded measurable functions on $\Omega$ in $L_{\Phi}(\Omega)$. In particular, $E_{\Phi}(\Omega)$ is separable and $\left(E_{\Phi}(\Omega)\right)^{*}=L_{\Psi}(\Omega)$. Therefore, we can extract a subsequence such that

$$
\mathrm{e}^{r_{k, N}} \rightarrow \mathrm{e}^{r_{k}} \quad \text { weakly* in } L^{\infty}(0, T ; L \log L(\Omega)),
$$

where by weak* convergence we mean that $\left\langle\mathrm{e}^{r_{k, N}}, \phi\right\rangle \rightarrow\left\langle\mathrm{e}^{r_{k}}, \phi\right\rangle$ for each $\phi \in L^{1}\left(0, T ; E_{\Phi}(\Omega)\right)$. Moreover, by (24) we deduce that for all $\eta \in \mathcal{D}(\Omega)$ the functions $t \rightarrow \int_{\Omega} r_{k, N}(t) \eta \mathrm{d} x$ form a bounded equicontinuous sequence in $C[0, T]$. Hence, the weak* convergence of $r_{k, N}, \mathrm{e}^{r_{k, N}}$ may be improved, using the Arzelá-Ascoli theorem and the density of $\mathcal{D}(\Omega)$ in $L^{p}$ for $p \in[1, \infty)$ and in $E_{\Phi}(\Omega)$, to the following:

$$
\begin{gathered}
r_{k, N} \rightarrow r_{k} \quad \text { in } C\left([0, T] ; L_{\text {weak }}^{2}(\Omega)\right), \\
\mathrm{e}^{r_{k, N}} \rightarrow \mathrm{e}^{r_{k}} \quad \text { in } C\left([0, T] ; L \log L_{\text {weak }^{*}}(\Omega)\right),
\end{gathered}
$$

which gives sense to the initial conditions for $r_{k}$ and $\mathrm{e}^{r_{k}}$.

Finally, as $\mathrm{e}^{r_{k, N}}>0$ on $[0, T] \times \Omega, \frac{m_{j} \mathrm{e}^{r_{j, N}}}{\varrho_{N}}$ is bounded in $L^{\infty}((0, T) \times \Omega)$ and so, the pointwise convergence of $\mathrm{e}^{r_{k, N}}$ for any $k=1, \ldots, n$ implies that

$$
\frac{m_{j} \mathrm{e}^{r_{j, N}}}{\varrho_{N}} \rightarrow \frac{m_{j} \mathrm{e}^{r_{j}}}{\sum_{k=1}^{n} m_{k} \mathrm{e}^{r_{k}}} \quad \text { strongly in } L^{p}((0, T) \times \Omega), p<\infty .
$$

Therefore, we also get the convergence of $\mathcal{F}_{k, N}$. Summarizing, the result achieved in this section can be stated as follows.

Lemma 4. Let the assumptions of Theorem 1 be fulfilled and let $\varrho_{k, N}, k=1, \ldots, n$ be the unique solution to the approximate problem (22) constructed in Theorem 2. Then there exists a subsequence $N_{l} \rightarrow+\infty$ such that the limit functions $r_{k}=\lim _{N_{l} \rightarrow \infty} r_{k, N_{l}}$ satisfy system (19) in the sense of distributions.

\section{Estimates independent of $\delta$, passage to the limit $\delta \rightarrow 0$}

In what follows, we will denote by $r_{k, \delta}, k=1, \ldots, n$, the solution to the approximate problem (19), constructed in Subsection III B. The next step of the proof is to let $\delta \rightarrow 0^{+}$in order to eliminate the artificial time derivative as well as the $\delta$-dependent parabolic regularization in (19). To this aim, we first need to derive some uniform bounds sufficient to deduce compactness of the nonlinear terms, which is the subject of the present subsection. We start by proving the energy inequality. 
Lemma 5. Let $\delta, \epsilon>0$, then the solution to (19) enjoys the following estimate:

$$
\begin{aligned}
& \sup _{t \in(0, T)} \sum_{k=1}^{n}\left\|\left(\delta r_{k, \delta}^{2}+\mathrm{e}^{r_{k, \delta}} r_{k, \delta}\right)(t)\right\|_{L^{1}(\Omega)} \\
& +\sum_{k=1}^{n}\left\{\delta \int_{0}^{T}\left\|\nabla r_{k, \delta}\right\|_{L^{2}(\Omega)} \mathrm{d} t+\varepsilon \int_{0}^{T}\left\|\nabla \sqrt{\mathrm{e}^{r_{k, \delta}}}\right\|_{L^{2}(\Omega)}^{2} \mathrm{~d} t+\int_{0}^{T}\left\|\frac{\mathcal{F}_{k, \delta}}{\sqrt{m_{k} \mathrm{e}^{r_{k, \delta}}}}\right\|_{L^{2}(\Omega)}^{2} \mathrm{~d} t\right\} \leq c,
\end{aligned}
$$

for a constant $c$ that depends only on the initial data and $T$.

Proof. Due to the pointwise convergence of $r_{k, N}$ and $\mathrm{e}^{r_{k, N}}$, see (39) and (40), we have

$$
\int_{\Omega}\left(\delta \frac{r_{k, N}^{2}}{2}+\mathrm{e}^{r_{k, N}} r_{k, N}-\mathrm{e}^{r_{k, N}}\right) \mathrm{d} x \rightarrow \int_{\Omega}\left(\delta \frac{r_{k, \delta}^{2}}{2}+\mathrm{e}^{r_{k, \delta}} r_{k, \delta}-\mathrm{e}^{r_{k, \delta}}\right) \mathrm{d} x
$$

in the sense of distributions on $(0, T)$. Moreover, we know that $\nabla r_{k, N}$ converges to $\nabla r_{k, \delta}$ weakly in $L^{2}((0, T) \times \Omega)$, thus, due to lower semicontinuity of convex functions we have

$$
\int_{0}^{T} \int_{\Omega}\left|\nabla r_{k}\right|^{2} \mathrm{~d} x \mathrm{~d} t \leq \liminf _{N \rightarrow \infty} \int_{0}^{T} \int_{\Omega}\left|\nabla r_{k, N}\right|^{2} \mathrm{~d} x \mathrm{~d} t .
$$

The same argument can be also applied for the nonlinear terms $\nabla \sqrt{\mathrm{e}^{r_{k, N}}}$ and $\frac{\mathcal{F}_{k, N}}{\sqrt{m_{k} \mathrm{e}^{r_{k, N}}}}$.

Having obtained the uniform estimates, we can return to our original problem. We define the solution to (5) in the following way:

$$
\varrho_{k, \delta}:=m_{k} \mathrm{e}^{r_{k, \delta}}, \quad k=1, \ldots, n .
$$

Hence, (42) gives rise to the following estimate:

$$
\text { ess } \sup _{t \in(0, T)} \int_{\Omega}\left(\log p_{k, \delta}(t)\right)^{2} \mathrm{~d} x+\int_{0}^{T} \int_{\Omega}\left|\nabla \log p_{k, \delta}\right|^{2} \mathrm{~d} x \mathrm{~d} t \leq c(\delta),
$$

which is equivalent to positivity of partial densities

$$
\varrho_{k, \delta}>0 \quad \text { a.e. in }(0, T) \times \Omega, k=1, \ldots, n .
$$

We may now repeat arguments leading to (36). More precisely, as we can now test by any function from the space $L^{5}\left(0, T ; W^{1,5}(\Omega)\right)$, due to a similar argument as in (37) and (38) we control $\partial_{t} r_{k, \delta}$ and $\partial_{t}\left(\delta r_{k_{\delta}}+\mathrm{e}^{r_{k, \delta}}\right)$; thus we also control its difference. Hence, uniformly with respect to $\delta$ we have

$$
\delta\left\|\partial_{t} \log p_{k, \delta}\right\|_{L^{\frac{5}{4}}\left(0, T ; W^{\left.-1, \frac{5}{4}(\Omega)\right)}\right.}+\left\|\partial_{t} p_{k, \delta}\right\|_{L^{\frac{5}{4}}\left(0, T ; W^{\left.-1, \frac{5}{4}(\Omega)\right)}\right.} \leq c .
$$

From (42) it also follows that the sequence $\sqrt{p_{k, \delta}}$ is uniformly bounded in $L^{2}\left(0, T ; W^{1,2}(\Omega)\right)$. Therefore, using again the Aubin-Lions lemma, we show that $\varrho_{k, \delta}=p_{k, \delta} m_{k}$ converges to $\varrho_{k}$ for $\delta \rightarrow 0$ pointwisely on $(0, T) \times \Omega$. This together with the uniform estimates from (42) allows us to deduce the following convergences when $\delta \rightarrow 0$ :

$$
\begin{gathered}
\delta \log \frac{\varrho_{k, \delta}}{m_{k}} \rightarrow 0 \quad \text { strongly in } L^{\infty}\left(0, T ; L^{2}(\Omega)\right), \\
\delta \nabla \log \frac{\varrho_{k, \delta}}{m_{k}} \rightarrow 0 \quad \text { strongly in } L^{2}((0, T) \times \Omega), \\
\varrho_{k, \delta} \rightarrow \varrho_{k} \quad \text { strongly in } L^{q}((0, T) \times \Omega), q<\frac{5}{3}, \\
\nabla \varrho_{k, \delta} \rightarrow \nabla \varrho_{k} \quad \text { weakly in } L^{2}\left(0, T ; L \log L_{\text {weak }^{*}}(\Omega)\right) \cap L^{\frac{5}{4}}((0, T) \times \Omega), \\
\varrho_{k, \delta} \rightarrow \varrho_{k} \quad \text { in } C\left([0, T] ; L \log L_{\text {weak }^{*}}(\Omega)\right) .
\end{gathered}
$$

Moreover

$$
\begin{gathered}
\frac{\varrho_{j, \delta}}{\sum_{k=1}^{n} \varrho_{k, \delta}} \rightarrow \frac{\varrho_{j}}{\sum_{k=1}^{n} \varrho_{k}} \quad \text { strongly in } L^{p}((0, T) \times \Omega), p<\infty, \\
\varrho_{k} \geq 0 \quad \text { a.e. in }(0, T) \times \Omega, k=1, \ldots, n,
\end{gathered}
$$


and due to a similar argument as in Subsection III B we obtain that

$$
\mathcal{F}_{k, \delta} \rightarrow \mathcal{F}_{k} \quad \text { weakly in } L^{p}((0, T) \times(\Omega)),
$$

for some $p>1$, where $\mathcal{F}_{k}$ depends on the limit functions $\varrho_{1}, \ldots, \varrho_{n}$ as specified in (7).

This is the last argument in favor to let $\delta \rightarrow 0$ in the approximate system (19), we have thus proved the following result.

Lemma 6. The limit quantities $\varrho_{k}, k=1, \ldots, n$ satisfy

$$
\partial_{t} \varrho_{k}+\operatorname{div}\left(\varrho_{k} \mathbf{u}\right)-\varepsilon \Delta \varrho_{k}+\operatorname{div}\left(\mathcal{F}_{k}\right)=\varrho \omega_{k}, \quad k=1, \ldots n,
$$

in the sense of distributions on $(0, T) \times \Omega$.

In addition, denoting $\varrho=\sum_{k=1}^{n} \varrho_{k}$, and summing (46) with respect to $k=1, \ldots, n$, the properties (13) and (15) lead to the following equation:

$$
\partial_{t} \varrho+\operatorname{div}(\varrho \mathbf{u})-\varepsilon \Delta \varrho=0 .
$$

This equation is, due to the previous lemma, satisfied in the same sense as system (46), together with the initial condition $\varrho(0, x)=\varrho^{0}=\sum_{k=1}^{n} \varrho_{k}^{0}(x)$ for a.a. $x \in \Omega$.

On the other hand, provided $\mathbf{u}$ and the initial data are sufficiently smooth (as in Theorem 1 is enough), we can identify $\varrho$ with $\varrho_{\varepsilon}$ - the unique classical positive solution to the initial-value problem

$$
\partial_{t} \varrho_{\varepsilon}+\operatorname{div}\left(\varrho_{\varepsilon} \mathbf{u}\right)-\varepsilon \Delta \varrho_{\varepsilon}=0, \quad \varrho_{\varepsilon}(0, x)=\varrho^{0}
$$

constructed by means of the usual Galerkin approach within the standard $L^{2}$ theory, the bootstrap argument and the maximal $L^{p}-L^{q}$ regularity applied to the problem

$$
\partial_{t} \varrho_{\varepsilon}-\varepsilon \Delta \varrho_{\varepsilon}=f:=-\operatorname{div}\left(\varrho_{\varepsilon} \mathbf{u}\right), \quad \varrho_{\varepsilon}(0, x)=\varrho^{0},
$$

see Secs. 7.6.3 - 7.6.7 of Ref. 24 for more details. In particular, we know that any solution of (46) satisfies

$$
\sum_{k=1}^{n} \varrho_{k}=\varrho_{\varepsilon} \quad \text { a.e. in }(0, T) \times \Omega,
$$

where $\varrho_{\epsilon}$ is the unique solution to (47).

\section{Estimates independent of $\varepsilon$}

This part of the proof is dedicated to derivation of estimates independent of $\varepsilon$ and to the last limit passage $\varepsilon \rightarrow 0$. The departure point is an analogue of (42),

$$
\sup _{t \in(0, T)} \sum_{k=1}^{n}\left\|p_{k, \varepsilon} \log p_{k, \varepsilon}(t)\right\|_{L^{1}(\Omega)}+\sum_{k=1}^{n}\left\{\varepsilon \int_{\Omega}\left\|\nabla \sqrt{p_{k, \varepsilon}}\right\|_{L^{2}(\Omega)}^{2} \mathrm{~d} x+\int_{0}^{T}\left\|\frac{\mathcal{F}_{k, \varepsilon}}{\sqrt{\varrho_{k, \varepsilon}}}\right\|_{L^{2}(\Omega)}^{2} \mathrm{~d} t\right\} \leq c,
$$

where the constant $c$ does not depend on $\epsilon$, and the fact that $Y_{k, \varepsilon}=\frac{\varrho_{k, \varepsilon}}{\varrho_{\varepsilon}}, k=1, \ldots, n$ satisfy

$$
0 \leq Y_{k, \varepsilon} \leq 1, \quad \sum_{k=1}^{n} Y_{k, \varepsilon}=1 .
$$

As we see, it is not so clear whether we have any additional space regularity of solutions. Indeed, to repeat the arguments from the previous limit passage, one needs to show that the quantities $\nabla \sqrt{\varrho_{k, \varepsilon}}$, $k=1, \ldots, n$ are controllable independently of $\varepsilon$. To this end, we investigate more carefully the last term in (49). We have the following result.

Lemma 7. Let assumptions of Theorem 1 be fulfilled and let estimate (49) be valid. Then, for any $k=1, \ldots, n$ the solution to the approximate problem (46) satisfies

$$
\left\|\nabla \sqrt{\varrho_{k, \varepsilon}}\right\|_{L^{2}((0, T) \times \Omega)} \leq c,
$$

where the constant $c$ does not depend on $\varepsilon$. 
Proof. Using (18) we deduce from (49) that

$$
\sum_{k=1}^{n} \int_{0}^{T} \int_{\Omega} \frac{\mathcal{F}_{k, \varepsilon}^{2}}{\varrho_{k, \varepsilon}} \mathrm{d} x \mathrm{~d} t=\sum_{k=1}^{n} \int_{0}^{T} \int_{\Omega} \frac{\left(\pi_{\varepsilon} \mathbf{d}_{k, \varepsilon}\right)^{2}}{\varrho_{k, \varepsilon}} \mathrm{d} x \mathrm{~d} t \leq c .
$$

To exploit this estimate we first recall the following property of the species diffusion forces:

$$
\pi \vec{d}_{\varepsilon}^{i}=\pi\left(\begin{array}{c}
d_{1, \varepsilon}^{i} \\
d_{2, \varepsilon}^{i} \\
\vdots \\
d_{n, \varepsilon}^{i}
\end{array}\right)=\left(\begin{array}{cccc}
Z_{1, \varepsilon} & -Y_{1, \varepsilon} & \ldots & -Y_{1, \varepsilon} \\
-Y_{2, \varepsilon} & Z_{2, \varepsilon} & \ldots & -Y_{2, \varepsilon} \\
\vdots & \vdots & \ddots & \vdots \\
-Y_{n, \varepsilon} & -Y_{n, \varepsilon} & \ldots & Z_{n, \varepsilon}
\end{array}\right) \cdot\left(\begin{array}{c}
\nabla_{x_{i}} p_{1, \varepsilon} \\
\nabla_{x_{i}} p_{2, \varepsilon} \\
\vdots \\
\nabla_{x_{i}} p_{n, \varepsilon}
\end{array}\right)=\mathbf{C}_{\varepsilon} \nabla_{x_{i}} \vec{p}_{\varepsilon}
$$

where $\vec{p}_{\varepsilon}$ denotes the $n$ dimensional vector $\left(p_{1, \varepsilon}, \ldots, p_{n, \varepsilon}\right)^{T}$ and $\nabla \vec{p}_{\varepsilon}=\left(\nabla p_{1, \varepsilon}, \ldots, \nabla p_{n, \varepsilon}\right)^{T}$.

So, inserting it to (51) we obtain

$$
\int_{0}^{T} \int_{\Omega} \sum_{k=1}^{n} \frac{\left(\mathbf{C}_{\varepsilon} \nabla \vec{p}_{\varepsilon}\right)_{k}^{2}}{\varrho_{k, \varepsilon}} \mathrm{d} x \mathrm{~d} t \leq c
$$

The matrix $\mathbf{C}_{\varepsilon}$ is degenerated as the vector $\vec{Y}_{\varepsilon}=\left(Y_{1, \varepsilon}, \ldots, Y_{n, \varepsilon}\right)^{T}$ belongs to its kernel, so estimate (53) does not imply integrability of $\nabla p_{k, \varepsilon}$ for all $k=1, \ldots, n$, at the same time. However, as we know that $\sum_{k=1}^{n} \varrho_{k, \varepsilon}$ coincides with the classical unique solution to (47) $\varrho_{\varepsilon}$, we can use the assumption on higher regularity of $\varrho_{\varepsilon}$ to control the full gradient of $\vec{p}_{\varepsilon}$. Indeed, note that the matrix $\mathbf{C}_{\varepsilon}$ possesses $n$ -1 eigenvectors $\vec{v}^{m}=\left(v_{1}^{m}, \ldots, v_{n}^{m}\right)^{t}, m \in\{1, \ldots, n-1\}$,

$$
v_{l}^{m}=\left\{\begin{aligned}
-1 & \text { for } \quad l=m, \\
1 & \text { for } \quad l=n, \\
0 & \text { for } \quad l \neq m, n,
\end{aligned}\right.
$$

corresponding to the eigenvalue 1 . Therefore, denoting

$$
\mathbf{C}_{\varepsilon}(t, x) \nabla_{x_{i}} \vec{p}_{\varepsilon}(t, x):=\left(\nabla_{x_{i}} \vec{p}_{\varepsilon}(t, x)\right)^{I}, \quad \text { for }(t, x) \in[0, T) \times \Omega,
$$

we have, for every $(k, i)$-th coordinate of $\nabla \vec{p}_{\varepsilon}(t, x),(k, i) \in\{1, \ldots, n\} \times\{1,2,3\}$, the following decomposition:

$$
\left(\nabla_{x_{i}} \vec{p}_{\varepsilon}(t, x)\right)_{k}=\left(\nabla_{x_{i}} \vec{p}_{\varepsilon}(t, x)\right)_{k}^{I}+\alpha_{i}(t, x) Y_{k, \varepsilon}(t, x),
$$

where $\alpha_{i}(t, x) Y_{k, \epsilon}(t, x)$ is the $k$ th coordinate of the projection of vector $\nabla_{x_{i}} \vec{p}_{\varepsilon}(t, x) \in \mathbb{R}^{n}$ on the nullspace of matrix $\mathbf{C}_{\varepsilon}(t, x)$, which is spanned by the vector $\vec{Y}_{\varepsilon}(t, x)$. Next, multiplying (54) by $m_{k}$ and summing over $k \in\{1, \ldots, n\}$ one gets

$$
\alpha_{i}=\frac{\nabla_{x_{i}} \varrho_{\varepsilon}}{\sum_{k=1}^{n} m_{k} Y_{k, \varepsilon}}-\frac{\sum_{k=1}^{n} m_{k}\left(\nabla_{x_{i}} \vec{p}_{\varepsilon}\right)_{k}^{I}}{\sum_{k=1}^{n} m_{k} Y_{k, \varepsilon}} .
$$

Combining this with (54) we can express each of the gradients of partial pressures in terms of known quantities

$$
\nabla_{x_{i}} \vec{p}_{\varepsilon}=\left(\nabla_{x_{i}} \vec{p}_{\varepsilon}\right)^{I}+\left(\frac{\nabla_{x_{i}} \varrho_{\varepsilon}}{\sum_{k=1}^{n} m_{k} Y_{k, \varepsilon}}-\frac{\sum_{k=1}^{n} m_{k}\left(\nabla_{x_{i}} \vec{p}_{\varepsilon}\right)_{k}^{I}}{\sum_{k=1}^{n} m_{k} Y_{k, \varepsilon}}\right) \vec{Y}_{\varepsilon} .
$$

Next, due to the first equality in (18) we can write

$$
\frac{\mathcal{F}_{k, \varepsilon}^{2}}{\varrho_{k, \varepsilon}}=\frac{\left|\nabla p_{k, \varepsilon}\right|^{2}}{\varrho_{k, \varepsilon}}-2 \frac{Y_{k, \varepsilon} \nabla p_{k, \varepsilon} \cdot \nabla \pi_{\varepsilon}}{\varrho_{k, \varepsilon}}+\frac{Y_{k, \varepsilon}^{2}\left|\nabla \pi_{\varepsilon}\right|^{2}}{\varrho_{k, \varepsilon}},
$$


which is bounded $L^{1}((0, T) \times \Omega)$ for every $k=1, \ldots, n$, on account of (51). Therefore, by the Cauchy inequality,

$$
\int_{0}^{T} \int_{\Omega} \frac{\left|\nabla p_{k, \varepsilon}\right|^{2}}{\varrho_{k, \varepsilon}} \mathrm{d} x \mathrm{~d} t \leq c\left(1+\int_{0}^{T} \int_{\Omega} \frac{Y_{k, \varepsilon}^{2}\left|\nabla \pi_{\varepsilon}\right|^{2}}{\varrho_{k, \varepsilon}} \mathrm{d} x \mathrm{~d} t\right) .
$$

Since $\nabla \pi_{\varepsilon}=\sum_{k=1}^{n}\left(\nabla \vec{p}_{\varepsilon}\right)_{k}$ and $\sum_{k=1}^{n}\left(\vec{Y}_{\varepsilon}\right)_{k}=1$, we can use (55) and (53) to estimate the right hand side of the above inequality. We have

$$
\begin{aligned}
& \int_{0}^{T} \int_{\Omega} \frac{Y_{k, \varepsilon}^{2}\left|\nabla \pi_{\varepsilon}\right|^{2}}{\varrho_{k, \varepsilon}} \mathrm{d} x \mathrm{~d} t=\int_{0}^{T} \int_{\Omega} \frac{Y_{k, \varepsilon}^{2}\left|\sum_{k=1}^{n}\left(\nabla \vec{p}_{\varepsilon}\right)_{k}\right|^{2}}{\varrho_{k, \varepsilon}} \mathrm{d} x \mathrm{~d} t \\
\leq & \int_{0}^{T} \int_{\Omega} \frac{Y_{k, \varepsilon}^{2}\left|\sum_{k=1}^{n}\left(\nabla \vec{p}_{\varepsilon}\right)_{k}^{I}\right|^{2}}{\varrho_{k, \varepsilon}} \mathrm{d} x \mathrm{~d} t+\int_{0}^{T} \int_{\Omega} \frac{Y_{k, \varepsilon}^{2}}{\varrho_{k, \varepsilon}}\left(\frac{\left|\nabla \varrho_{\varepsilon}\right|^{2}}{\left(\sum_{k=1}^{n} m_{k} Y_{k, \varepsilon}\right)^{2}}+\frac{\left|\sum_{k=1}^{n} m_{k}\left(\nabla \vec{p}_{\varepsilon}\right)_{k}^{I}\right|^{2}}{\left(\sum_{k=1}^{n} m_{k} Y_{k, \varepsilon}\right)^{2}}\right) \mathrm{d} x \mathrm{~d} t \\
= & \int_{0}^{T} \int_{\Omega} \frac{Y_{k, \varepsilon}^{2}}{\varrho_{k, \varepsilon}}\left(\left|\sum_{k=1}^{n}\left(\nabla \vec{p}_{\varepsilon}\right)_{k}^{I}\right|^{2}+\frac{\left|\sum_{k=1}^{n} m_{k}\left(\nabla \vec{p}_{\varepsilon}\right)_{k}^{I}\right|^{2}}{\left(\sum_{k=1}^{n} m_{k} Y_{k, \varepsilon}\right)^{2}}\right) \mathrm{d} x \mathrm{~d} t+\int_{0}^{T} \int_{\Omega} \frac{Y_{k, \varepsilon}^{2}}{\varrho_{k, \varepsilon}} \frac{\left|\nabla \varrho_{\varepsilon}\right|^{2}}{\left(\sum_{k=1}^{n} m_{k} Y_{k, \varepsilon}\right)^{2}} \mathrm{~d} x \mathrm{~d} t .
\end{aligned}
$$

Next, denoting $m_{\max }=\max \left\{m_{1}, \ldots, m_{n}\right\}$ and $m_{\min }=\min \left\{m_{1}, \ldots, m_{n}\right\}$, the first term from the right hand side of (57) can be estimated as follows:

$$
\begin{gathered}
\int_{0}^{T} \int_{\Omega} \frac{Y_{k, \varepsilon}^{2}}{\varrho_{k, \varepsilon}}\left(\left|\sum_{k=1}^{n}\left(\nabla \vec{p}_{\varepsilon}\right)_{k}^{I}\right|^{2}+\frac{\left|\sum_{k=1}^{n} m_{k}\left(\nabla \vec{p}_{\varepsilon}\right)_{k}^{I}\right|^{2}}{\left(\sum_{k=1}^{n} m_{k} Y_{k, \varepsilon}\right)^{2}}\right) \mathrm{d} x \mathrm{~d} t \\
\quad \leq \int_{0}^{T} \int_{\Omega} \frac{Y_{k, \varepsilon}}{\varrho_{\varepsilon}}\left(\sum_{k=1}^{n}\left|\left(\nabla \vec{p}_{\varepsilon}\right)_{k}^{I}\right|^{2}+\frac{m_{\max }^{2} \sum_{k=1}^{n}\left|\left(\nabla \vec{p}_{\varepsilon}\right)_{k}^{I}\right|^{2}}{m_{\min }^{2}}\right) \mathrm{d} x \mathrm{~d} t \\
\leq \int_{0}^{T} \int_{\Omega} \frac{Y_{k, \varepsilon}}{\varrho_{\varepsilon}}\left(1+\frac{m_{\max }^{2}}{m_{\min }^{2}}\right) \sum_{k=1}^{n} \frac{\varrho_{k, \varepsilon}\left|\left(\nabla \vec{p}_{\varepsilon}\right)_{k}^{I}\right|^{2}}{\varrho_{k, \varepsilon}} \mathrm{d} x \mathrm{~d} t \leq c \int_{0}^{T} \int_{\Omega} \sum_{k=1}^{n} \frac{\left|\left(\nabla \vec{p}_{\varepsilon}\right)_{k}^{I}\right|^{2}}{\varrho_{k, \varepsilon}} \mathrm{d} x \mathrm{~d} t,
\end{gathered}
$$

which is bounded due to (53).

The second integral on the right hand side of (57) can be bounded since $Y_{k, \varepsilon} \leq 1$ for any $k=1, \ldots, n$,

$$
\int_{0}^{T} \int_{\Omega} \frac{Y_{k, \varepsilon}^{2}}{\varrho_{k, \varepsilon}} \frac{\left|\nabla \varrho_{\varepsilon}\right|^{2}}{\left(\sum_{k=1}^{n} m_{k} Y_{k, \varepsilon}\right)^{2}} \mathrm{~d} x \mathrm{~d} t \leq c \int_{0}^{T} \int_{\Omega} \frac{\left|\nabla \varrho_{\varepsilon}\right|^{2}}{\varrho_{\varepsilon}} \mathrm{d} x \mathrm{~d} t .
$$

Returning to (56), we have thus shown that

$$
\int_{0}^{T} \int_{\Omega} \frac{\left|\nabla p_{k, \varepsilon}\right|^{2}}{\varrho_{k, \varepsilon}} \mathrm{d} x \mathrm{~d} t \leq c\left(1+\int_{0}^{T} \int_{\Omega} \frac{\left|\nabla \varrho_{\varepsilon}\right|^{2}}{\varrho_{\varepsilon}} \mathrm{d} x \mathrm{~d} t\right) .
$$

Using Lemma 8 below we control $\nabla \varrho_{\epsilon}$ in $L^{2}((0, T) \times \Omega)$ independently of $\varepsilon$; due to the properties of the initial value (strict positivity) we therefore also control $\nabla \sqrt{\varrho_{\varepsilon}}$ in the same space. The proof of the lemma is finished.

Lemma 8. Under the assumptions of Theorem 1, there exists $c$ independent of $\epsilon$ such that for any $\epsilon \in(0,1]$,

$$
\left\|\varrho-\varrho_{\varepsilon}\right\|_{L^{2}\left(0, T ; W^{1,2}(\Omega)\right)} \leq c,
$$

where $\varrho$ is the unique solution to (3) and $\varrho_{\epsilon}$ is the unique solution to (47). 
Proof. We have

$$
\partial_{t}\left(\varrho_{\varepsilon}-\varrho\right)+\operatorname{div}\left(\boldsymbol{u}\left(\varrho_{\varepsilon}-\varrho\right)\right)-\varepsilon \Delta\left(\varrho_{\varepsilon}-\varrho\right)=\varepsilon \Delta \varrho,
$$

with $\left(\varrho_{\varepsilon}-\varrho\right)(0, x)=0$. Testing equation above by $\varrho_{\varepsilon}-\varrho$ and recalling that $\|\varrho\|_{L^{2}\left(0, T ; W^{1,2}(\Omega)\right)}<\infty$, we get the result.

This is the final argument that allows us to repeat the procedure described for the limit passage $\delta \rightarrow 0$ in order to eliminate the last regularizing term from (46). The proof of Theorem 1 is now complete.

\section{ACKNOWLEDGMENTS}

The work of P.B.M. and E.Z. has been supported by Polish MN Grant No. IdP2011 000661 and by the Foundation for Polish Science in framework of European Regional Development Funds (OPIE 2007-2013). The work of M.P. was supported by the Grant Agency of the Czech Republic No. 201/09/0917.

${ }^{1}$ R. A. Adams and J. J. F. Fournier, Sobolev Spaces, 2nd ed. (Elsevier, Amsterdam, 2003).

${ }^{2}$ H. Alt and S. Luckhaus, "Quasilinear elliptic-parabolic differential equations," Math. Z. 183, 311-341 (1983).

${ }^{3}$ J. Bebernes and D. Eberly, Mathematical Problems from Combustion Theory, Applied Mathematical Sciences Vol. 83 (Springer-Verlag, New York, 1989).

${ }^{4}$ D. Bothe, "On the Maxwell-Stefan approach to multicomponent diffusion," Progress in Nonlinear Differential Equations and their Applications, pp. 81-93. Springer, Basel, 2011.

${ }^{5}$ D. Bresch and B. Desjardins, "On the existence of global weak solutions to the Navier-Stokes equations for viscous compressible and heat conducting fluids," J. Math. Pures Appl. 87(1), 57-90 (2007).

${ }^{6}$ D. Bresch, B. Desjardins, and C.-K. Lin, "On some compressible fluid models: Korteweg, lubrication, and shallow water systems," Commun. Partial Differ. Equ. 28(3-4), 843-868 (2003).

${ }^{7}$ J. A. Carrillo, A. Jüngel, P. A. Markowich, G. Toscani, and A. Unterreiter, "Entropy dissipation methods for degenerate parabolic problems and generalized Sobolev inequalities," Monatsh. Math. 133(1), 1-82 (2001).

${ }^{8}$ G.-Q. Chen, D. Hoff, and K. Trivisa, "Global solutions to a model for exothermically reacting, compressible flows with large discontinuous initial data," Arch. Ration. Mech. Anal. 166(4), 321-358 (2003).

${ }^{9}$ L. Chen and A. Jüngel, "Analysis of a multidimensional parabolic population model with strong cross-diffusion," SIAM J. Math. Anal. 36(1), 301-322 (2004).

${ }^{10}$ P. Degond, S. Génieys, and A. Jüngel, "A system of parabolic equations in nonequilibrium thermodynamics including thermal and electrical effects," J. Math. Pures Appl. 76(10), 991-1015 (1997).

${ }^{11}$ D. Donatelli and K. Trivisa, "On the motion of a viscous compressible radiative-reacting gas," Commun. Math. Phys. 265(2), 463-491 (2006).

${ }^{12}$ B. Ducomet, "A model of thermal dissipation for a one-dimensional viscous reactive and radiative gas," Math. Methods Appl. Sci. 22(15), 1323-1349 (1999).

${ }^{13}$ E. Feireisl and A. Novotný, Singular Limits in Thermodynamics of Viscous Fluids, Advances in Mathematical Fluid Mechanics (Birkhäuser Verlag, Basel, 2009).

${ }^{14}$ E. Feireisl, H. Petzeltová, and K. Trivisa, "Multicomponent reactive flows: Global-in-time existence for large data," Commun. Pure Appl. Anal. 7(5), 1017-1047 (2008).

${ }^{15}$ H. Gajewski and K. Zacharias, "Global behaviour of a reaction-diffusion system modelling chemotaxis," Math. Nachr. 195, 77-114 (1998).

${ }^{16}$ V. Giovangigli, Multicomponent Flow Modeling, Modeling and Simulation in Science, Engineering and Technology (Birkhäuser Boston Inc., Boston, MA, 1999).

${ }^{17}$ R. Klein, N. Botta, T. Schneider, C. D. Munz, S. Roller, A. Meister, L. Hoffmann, and T. Sonar, "Asymptotic adaptive methods for multi-scale problems in fluid mechanics," J. Eng. Math. 39(1-4), 261-343 (2001).

${ }^{18}$ A. Kufner, O. John, and S. Fučik, Function Spaces (Academia, Praha, 1977).

${ }^{19}$ M. Lewicka and P. B. Mucha, "On temporal asymptotics for the $p$ th power viscous reactive gas," Nonlinear Anal. 57(7-8), 951-969 (2004).

${ }^{20}$ J. Málek and K. R. Rajagopal, “A thermodynamic framework for a mixture of two liquids," Nonlinear Anal.: Real World Appl. 9(4), 1649-1660 (2008).

${ }^{21}$ A. Mellet and A. Vasseur, "On the barotropic compressible Navier-Stokes equations," Commun. Partial Differ. Equ. 32(1-3), 431-452 (2007).

${ }^{22}$ P. B. Mucha and M. Pokorný, "On the steady compressible Navier-Stokes-Fourier system,” Commun. Math. Phys. 288(1), 349-377 (2009).

${ }^{23}$ A. Novotný and M. Pokorný, "Weak and variational solutions to steady equations for compressible heat conducting fluids," SIAM J. Math. Anal. 43(3), 1158-1188 (2011).

${ }^{24}$ A. Novotný and I. Straškraba, Introduction to the Mathematical Theory of Compressible Flow, Oxford Lecture Series in Mathematics and its Applications Vol. 27 (Oxford University Press, Oxford, 2004). 
${ }^{25}$ B. Perthame, "Transport equations in biology, Frontiers in Mathematics (Birkhäuser Verlag, Basel, 2007).

${ }^{26}$ T. Roubíček, "Incompressible ionized fluid mixtures," Continuum Mech. Thermodyn. 17(7), 493-509 (2006).

${ }^{27}$ L. Waldmann and E. Trübenbacher, "Formale kinetische theorie von Gasgemischen aus anregbaren Molekülen," Z. Naturforsch 17A, 363-376 (1962).

${ }^{28}$ E. Zatorska, "On a steady flow of multicomponent, compressible, chemically reacting gas," Nonlinearity 24, 3267-3278 (2011).

${ }^{29}$ E. Zatorska, “On the flow of chemically reacting gaseous mixture," J. Differ. Equations 253(12), 3471-3500 (2012). 